01

\title{
Формирование композиционных хаотических мультиаттракторов, содержащих неоднородности
}

\author{
(C) В.Г. Прокопенко
}

Южный федеральный университет, 344006 Ростов-на-Дону, Россия

e-mail: vadipro@yandex.ru

(Поступило в Редакцию 3 октября 2016 г.)

Представлены два способа введения неоднородностей в композиционные хаотические мультиаттракторы. Первый способ позволяет менять взаимное расположение элементов мультиаттрактора. Второй способ дает возможность задавать различия между хаотическими аттракторами, образующими мультиаттрактор.

DOI: $10.21883 / J T F .2017 .08 .44717 .2057 n$

\section{Введение}

Нестационарность и дискретность многих физических объектов и процессов, а также широкое применение методов системного анализа иногда порождают динамические модели, эволюция которых характеризуется случайной сменой множества положений равновесия, каждое из которых, в свою очередь, представляет собой область нестационарного поведения - хаотический аттрактор [1-5], т.е. движение происходит на составном (композиционном) хаотическом мультиаттракторе [6]. При этом для адекватного описания моделируемого объекта, как правило, необходимо, чтобы образующие мультиаттрактор элементы - локальные хаотические аттракторы - могли различаться между собой, а структуру мультиаттрактора можно было менять.

Однако разработанные к настоящему времени способы построения динамических систем, имеющих такие области равновесия, позволяют получать только однородные мультиаттракторы, имеющие фиксированную структуру и состоящие из одинаковых хаотических аттракторов, являющихся точной копией аттрактора некоторой исходной динамической системы [6-12].

В случае конечномерных динамических систем движение на однородном композиционном хаотическом мультиаттракторе в общем случае описывает следующая система обыкновенных дифференциальных уравнений с постоянными коэффициентами [6]:

$$
\dot{x}=\alpha H(x)+f[x, \eta]
$$

где

$$
\begin{gathered}
\dot{x}=\left[\begin{array}{c}
\dot{x}_{1} \\
\dot{x}_{2} \\
\ldots \\
\dot{x}_{n}
\end{array}\right], \quad \dot{x}_{j}=\frac{d x_{j}}{d t}, \quad j=1,2, \ldots, n ; \\
H(x)=\left[\begin{array}{c}
H_{1}\left(x_{1}\right) \\
H_{2}\left(x_{2}\right) \\
\ldots \\
H_{n}\left(x_{n}\right)
\end{array}\right], \quad \alpha=\left[\begin{array}{cccc}
\alpha_{11} & \alpha_{12} & \ldots & \alpha_{1 n} \\
\alpha_{21} & \alpha_{22} & \ldots & \alpha_{2 n} \\
\ldots & \ldots & \ldots & \ldots \\
\alpha_{n 1} & \alpha_{n 2} & \ldots & \alpha_{n n}
\end{array}\right],
\end{gathered}
$$

$$
\begin{aligned}
& f(x, \eta)= \\
& =\left[\begin{array}{c}
f_{1}\left[H_{1}\left(x_{1}\right), H_{2}\left(x_{2}\right), \ldots, H_{n}\left(x_{n}\right), \eta_{11}, \eta_{12}, \ldots \eta_{1 m_{1}}\right] \\
f_{2}\left[H_{1}\left(x_{1}\right), H_{2}\left(x_{2}\right), \ldots, H_{n}\left(x_{n}\right), \eta_{21}, \eta_{22}, \ldots \eta_{2 m_{2}}\right] \\
\ldots \\
f_{n}\left[H_{1}\left(x_{1}\right), H_{2}\left(x_{2}\right), \ldots, H_{n}\left(x_{n}\right), \eta_{n 1}, \eta_{n 2}, \ldots \eta_{n m_{n}}\right]
\end{array}\right],
\end{aligned}
$$

Здесь $x=x_{1}, x_{2}, \ldots x_{n}$ - так называемые переменные репликации [6], каждая из которых представляет собой либо независимую переменную, либо линейную комбинацию независимых переменных исходной динамической системы, $\alpha$ - константы линейной части уравнений, $f_{j}(\xi)$ - нелинейные члены, $\eta_{j}-$ константы, входящие в уравнения нелинейных членов, $H_{j}\left(x_{j}\right)$ - реплицирующие операторы, обеспечивающие образование копий хаотического аттрактора исходной динамической системы и их объединение в единый мультиаттрактор.

Реплицирующие (редуплицирующие, репликаторные) операторы (функции) представляют собой нелинейные функции переменных репликации, состоящие из линейных сегментов с единичным наклоном, соединенных между собой промежуточными участками (это могут быть линейные или нелинейные сегменты с противоположным наклоном, разрывы первого рода или области гистерезиса).

Реплицирующую функцию, состоящую из линейных сегментов, можно представить, например, следующим выражением [10]:

$$
\begin{aligned}
& H_{j}\left(x_{j}\right)=x_{j}+\left(d_{j}+1\right)\left\{P\left(x_{j}+s_{j}+h_{j}+\frac{h_{j}}{d_{j}}\right)\right. \\
& +P\left(x_{j}+s_{j}-h_{j}-\frac{h_{j}}{d_{j}}\right) \\
& -\sum_{m=0}^{M_{j}}\left[P\left(x_{j}+s_{j}-(2 m-1)\left(h_{j}+\frac{h_{j}}{d_{j}}\right)\right)+\frac{h_{j}}{d_{j}}\right] \\
& \left.-\sum_{n=0}^{N_{j}}\left[P\left(x_{j}+s_{j}+(2 n-1)\left(h_{j}+\frac{h_{j}}{d_{j}}\right)\right)-\frac{h_{j}}{d_{j}}\right]\right\},
\end{aligned}
$$




$$
P\left(x_{j}\right)=\frac{1}{2}\left(\left|x_{j}+\frac{h_{j}}{d_{j}}\right|-\left|x_{j}-\frac{h_{j}}{d_{j}}\right|\right),
$$

где $h_{j}-$ половина протяженности фазовой ячейки, содержащей хаотический аттрактор исходной динамической системы, по $j$-й переменной репликации, $M_{j}-$ количество локальных хаотических аттракторов вверх по $j$-й переменной репликации от исходного хаотического аттрактора, $N_{j}$ - количество локальных хаотических аттракторов вниз по $j$-й переменной репликации от исходного хаотического аттрактора, $d_{j}-$ модуль крутизны промежуточных сегментов реплицирующей функции по $j$-й переменной репликации, $s_{j}$ - константа, учитывающая асимметрию аттрактора исходной динамической системы по $j$-й переменной репликации.

От 0 до $n-1$ реплицирующих функций в уравнениях (1) может состоять из единственного сегмента с единичным наклоном, т. е. совпадать со своим аргументом. При наличии в системе (1) таких, „вырожденных“, операторов репликация будет осуществляться только по части переменных репликации [10-12].

Действие $m(m \leq n)$ невырожденных реплицирующих операторов на динамическую систему можно представить как формирование в фазовом пространстве этой системы (1) m-мерного массива фазовых ячеек, внутри каждой из которых находится фрагмент фазового пространства, идентичный фрагменту фазового пространства исходной динамической системы, содержащего ее аттрактор. Внутренние области фазовых ячеек соответствуют сегментам с единичным наклоном. Промежуточные сегменты реплицирующих функций отвечают слоям фазового пространства, разделяющим фазовые ячейки. Если все реплицирующие функции определены уравнением (2), то количество локальных аттракторов в составе мультиаттракторов составляет

$$
\prod_{j=1}^{m}\left(1+M_{j}+N_{j}\right) .
$$

Параметры реплицирующих функций выбираются так, чтобы границы фазовых ячеек пересекались с заключенными в них хаотическими аттракторами, отсекая от них малые периферийные области, а переменные репликации - так, чтобы каждая отсеченная область данного аттрактора принадлежала области притяжения хаотического аттрактора, расположенного в соседней фазовой ячейке. Двигаясь на данном хаотическом аттракторе, фазовая точка рано или поздно должна будет попасть в ту его часть, которая отсечена границей фазовой ячейки, т.е. пересечь границу фазовой ячейки. После этого она окажется в области притяжения соседнего аттрактора и перейдет на него. Поэтому движение на композиционном мультиаттракторе состоит из двух элементов автономных колебаний на локальных хаотических аттракторах и переходов с одного локального аттрактора на другой. Каждый переход на соседний аттрактор носит необратимый характер в том смысле, что фазовая точка после перехода на соседний хаотический аттрактор имеет шанс в дальнейшем перейти не только на предыдущий аттрактор, но и на любой другой соседний аттрактор [6].

Таким образом, колебания на каждом локальном аттракторе происходят независимо от наличия в составе мультиаттрактора всех остальных локальных аттракторов (следовательно, независимо и от их свойств). Также и выбор направлений переходов движения с одного локального хаотического аттрактора на другие никак не зависит от свойств локальных аттракторов, в частности от различий в эффективности преобразования энергии. Поэтому особенностью динамических систем рассматриваемого типа является отсутствие перераспределения движения в пользу тех или иных локальных аттракторов в зависимости от свойств движения на них, в частности от эффективности преобразования поступающей в систему энергии в энергию колебаний.

Это обусловливает возможность формирования неоднородных составных мультиаттракторов. В частности, имеющих перестраиваемую композиционную структуpy, а также состоящих из неодинаковых локальных аттракторов.

\section{Способ получения неоднородной композиционной структуры составного хаотического мультиаттрактора}

Известные в настоящее время способы построения хаотических мультиаттракторов позволяют получать мультиаттракторы, имеющие простейшую композиционную структуру, характеризующуюся тем, что локальные аттракторы образуют сплошной массив, не содержащий пустых позиций. Поэтому все разнообразие конфигураций таких мультиаттракторов ограничивается различиями в количестве элементов в составе мультиаттрактора и его положении относительно начала координат [10-13].

Например, „двумерный“ мультиаттрактор таких систем может иметь только конфигурации, аналогичные показанной на рис. 1, $a$. Но конфигурации, приведенные на рис. $1, b$, в известных динамических системах данного типа в настоящее время нереализуемы.

Это весьма существенно ограничивает возможности использования динамических систем, имеющих композиционные мультиаттракторы, как в плане формирования хаотических сигналов с заданным свойствами, так и в плане моделирования тех или иных физических процессов и объектов, имеющих множество связанных положений равновесия с неоднородной или изменяемой структурой.

Например, в автостохастической системе [11]

$$
\left\{\begin{array}{l}
\frac{d x}{d \tau}=A\left[H_{2}(y)-H_{1}(x)-z\right], \\
\frac{d y}{d \tau}=f(z)+H_{1}(x)-H_{2}(y)+z, \\
\frac{d z}{d \tau}=B\left[H_{1}(x)\right],
\end{array}\right.
$$



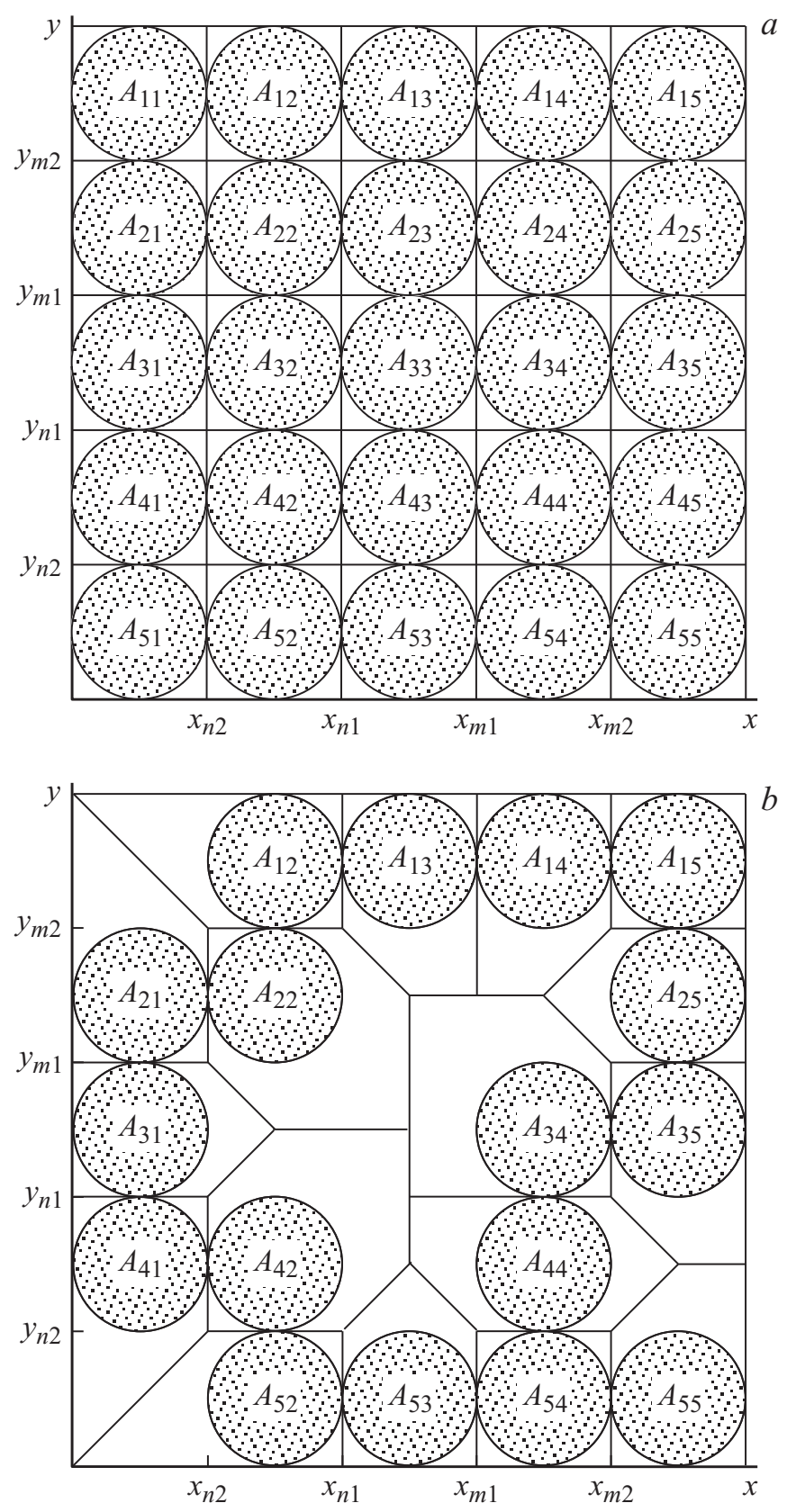

Рис. 1. Примеры различных вариантов структуры составного хаотического мультиаттрактора с предельной композиционной размерностью $5 \times 5$ элементов.

где $f(z)=b z+(a-b) \frac{|z+1|-|z-1|}{2} ; A, B, a, b-$ константы, функции $H_{1}(x)$ и $H_{2}(y)$ обеспечивают формирование мультиаттрактора, состоящего из $\left(1+M_{1}+\right.$ $\left.+N_{1}\right) \times\left(1+M_{2}+N_{2}\right)$ хаотических аттракторов, идентичных аттрактору исходной системы.

При использовании реплицирующих операторов вида (2) все возможные позиции в структуре мультиаттрактора системы (3) оказываются занятыми локальными аттракторами, т.е. для формирования заданной композиционной структуры необходима возможность удаления из состава мультиаттрактора части локальных аттракто- ров. Удалить локальный аттрактор можно только вместе с содержащей его ячейкой фазового пространства. Это можно сделать, расширив и сомкнув друг с другом граничащие с ней ячейки. В результате область фазового пространства, ранее содержавшей локальный аттрактор, будет заменена „пустыми“ областями соседних ячеек.

Например, при $M_{1}=N_{1}=M_{2}=N_{2}=2$ мультиаттрактор системы (1), (2) имеет композиционную структуру, показанную на рис. $1, a$. Чтобы получить, допустим, структуру, показанную на рис. $1, b$, нужно удалить локальные аттракторы $A_{11}, A_{23}, A_{24}, A_{32}, A_{33}, A_{43}, A_{45}$ и $A_{51}$. Эту задачу можно решить, расширив ячейки фазового пространства, содержащие локальные аттракторы $A_{12}, A_{13}, A_{14}, A_{21}, A_{22}, A_{25}, A_{31}, A_{34}, A_{35}, A_{41}, A_{42}, A_{44}$, $A_{52}$ и $A_{55}$, например, так, как показано на рис. $1, b$.

Так как формирование фазовых ячеек обеспечивают реплицирующие функции, изменение конфигурации ячеек требует соответствующего изменения уравнений этих функций. В них необходимо ввести члены, обеспечивающие смещение границ ячеек относительно исходного положения. Для этого уравнения (2) можно преобразовать к следующему виду:

$$
\begin{aligned}
& H_{k}\left(\xi_{k}, \xi_{\eta}\right)=\xi_{k}+\left(d_{k}+1\right)\left\{P\left(\xi_{k}+s_{k}+h_{k}+\frac{h_{k}}{d_{k}}-\Delta m\left(\xi_{\eta}\right)_{k, 0}\right)\right. \\
& +P\left(\xi_{k}+s_{k}-h_{k}-\frac{h_{k}}{d_{k}}-\Delta n\left(\xi_{\eta}\right)_{k, 0}\right) \\
& -\sum_{j=0}^{M_{k}}\left[P\left(\xi_{k}+s_{k}-(2 j-1)\left(h_{k}+\frac{h_{k}}{d_{k}}\right)\right)+\frac{h_{k}}{d_{k}}-\Delta m\left(\xi_{\eta}\right)_{k, j}\right] \\
& \left.-\sum_{j=0}^{N_{k}}\left[P\left(\xi_{k}+s_{k}+(2 j-1)\left(h_{k}+\frac{h_{k}}{d_{k}}\right)\right)-\frac{h_{k}}{d_{k}}-\Delta n\left(\xi_{\eta}\right)_{k, j}\right]\right\},
\end{aligned}
$$

где $\Delta m\left(\xi_{\eta}\right)_{k, j}, \Delta n\left(\xi_{\eta}\right)_{k, j}$ - члены, задающие отклонение границ расширяемых ячеек от их положения, определяемого уравнениями (2); $\xi_{\eta}=\xi_{\eta 1} \ldots \xi_{\eta L}-$ переменные, по которым производится смещение границ, $L-$ количество этих переменных.

Поэтому для формирования мультиаттрактора, имеющего композиционную структуру, показанную на рис. $1, b$, систему (3) необходимо переобразовать к виду

$$
\left\{\begin{array}{l}
\frac{d x}{d \tau}=A\left[H_{2}(y, x)-H_{1}(x, y)-z\right], \\
\frac{d y}{d \tau}=f(z)+H_{1}(x, y)-H_{2}(y, x)+z \\
\frac{d z}{d \tau}=B\left[H_{1}(x, y)\right] .
\end{array}\right.
$$

На рис. 2 и 3 приведены графики функций $\Delta m(y)_{1, j}$, $\Delta n(y)_{1, j}, \Delta m(x)_{2, j}, \Delta n(x)_{2, j}$, обеспечивающих формирование конфирурации фазовых ячеек, показанной на рис. $1, b$. 

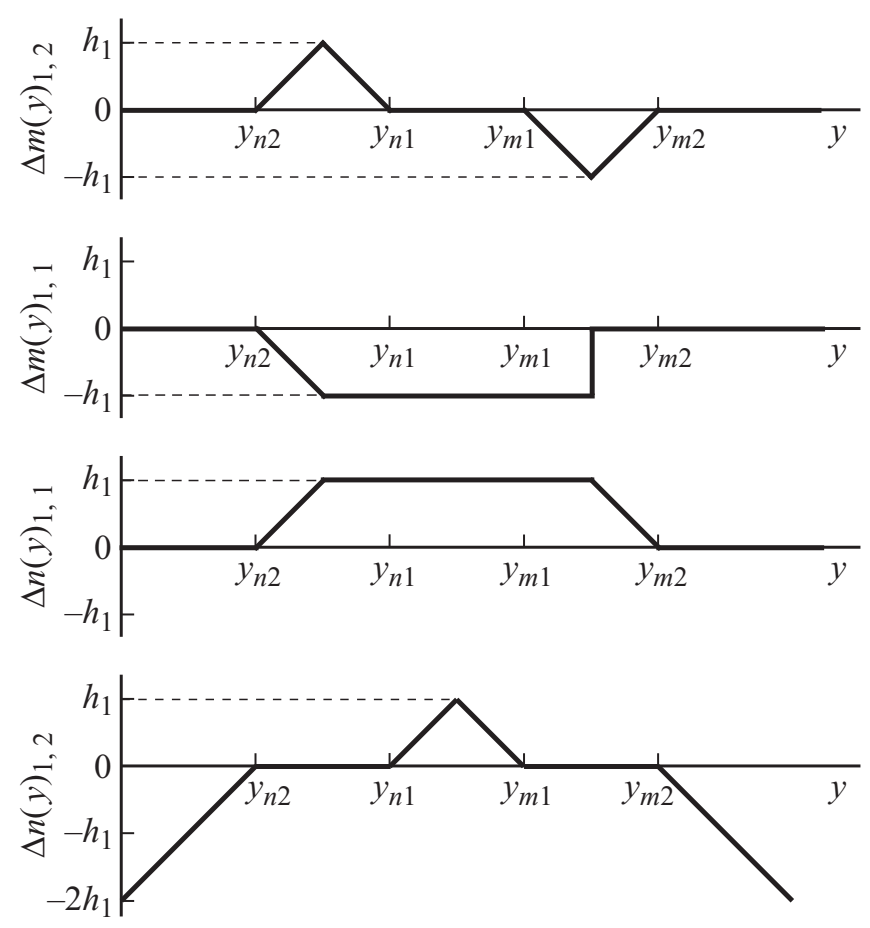

Рис. 2. Функции, задающие смещение границ между ячейками фазового пространства, формируемых реплицирующим оператором $H_{1}(x, y)$, необходимые для формирования показанной на рис. $1, b$ конфигурации фазовых ячеек.
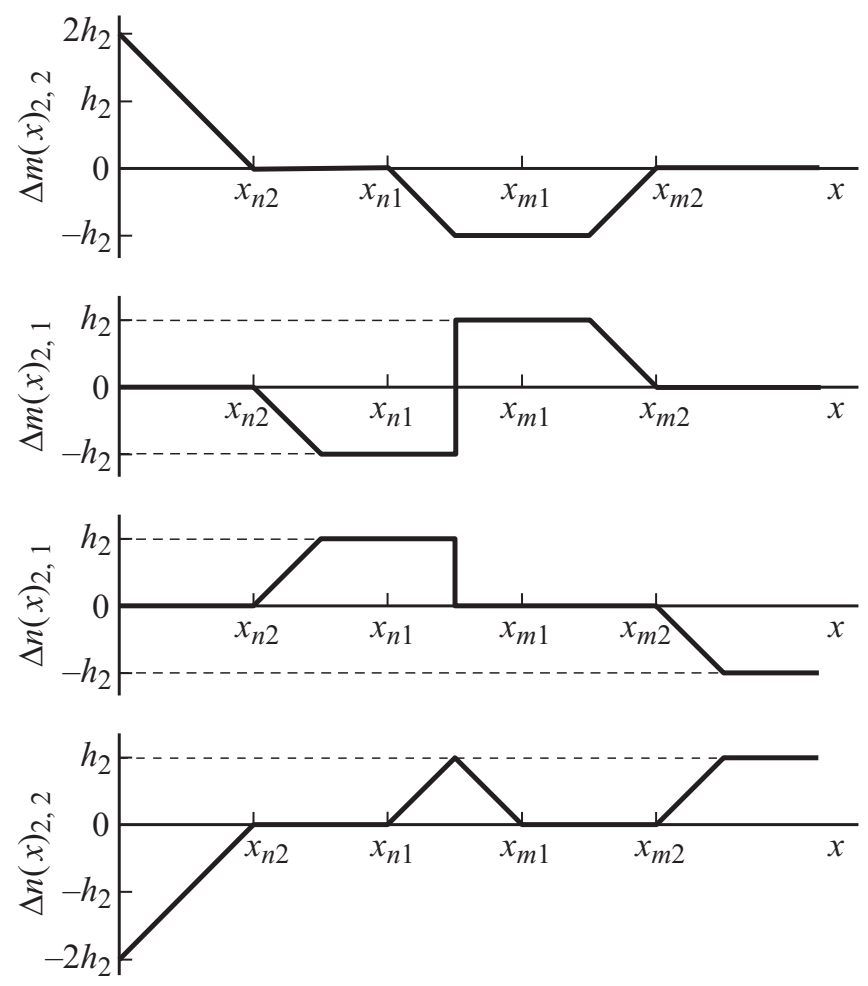

Рис. 3. Функции, задающие смещение границ между ячейками фазового пространства, формируемых реплицирующим оператором $H_{2}(y, x)$, необходимые для формирования показанной на рис. $1, b$ конфигурации фазовых ячеек.
В уравнениях (4) $\Delta m(y)_{1,0}=\Delta n(y)_{1,0}=\Delta m(x)_{2,0}=$ $=\Delta n(x)_{2,0}=0$. Остальные, отклоняющие функции (показанные на рис. 2 и 3) представляют собой кусочнолинейные зависимости, которые можно представить следующим выражением:

$$
\Delta(\xi)=\left\{\begin{array}{c}
V_{1} \xi+W_{1}, \quad \xi<\Xi_{1}, \\
V_{2} \xi+W_{2}, \quad \Xi_{1} \leq \xi<\Xi_{2}, \\
\ldots \ldots \ldots \ldots \ldots \ldots \ldots \ldots \\
V_{q} \xi+W_{q}, \quad \Xi_{q-1} \leq \xi<\Xi_{q}, \\
\ldots \ldots \ldots \ldots \ldots \ldots \ldots \\
V_{Q} \xi+W_{Q}, \quad \xi>\Xi_{Q-1},
\end{array}\right.
$$

где $Q-$ количество линейных сегментов в составе функции.

Параметры уравнения (6), соответствующие функциям на рис. 2 и 3 , приведены в табл. 1.

На рис. 4 изображен мультиаттрактор динамической системы (4)-(6), наблюдающийся при $A=0.5, B=1.9$, $a=20, b=-3, M_{1}=N_{1}=M_{2}=N_{2}=2, h_{1}=6.7, s_{1}=$ $=0, d_{1}=30, h_{2}=14.7, s_{2}=0, d_{2}=100$. Перестройка композиционной стуктуры мультиаттрактора осуществляется изменением отклоняющих функций, т.е. в конечном итоге сводится к изменению констант в уравнении (6).

\section{Способ построения составных хаотических аттракторов, состоящих из неодинаковых элементов}

Другой возможностью введения неоднородностей в композиционный хаотический мультиаттрактор является присвоение индивидуальных особенностей образующим его элементам - локальными хаотическими аттракторам.

Рассмотрим способ построения динамических систем, имеющих неоднородный композиционный хаотический мультиаттрактор, локальные хаотические аттракторы которого принадлежат одной и той же динамической системе, но отвечают разным значениям констант, входящих в уравнения движения.

Чтобы локальные аттракторы различались между собой, очевидно, должны различаться соответствующие им уравнения движения. В рассматриваемом случае для этого необходимо, чтобы значения констант в уравнениях (1) имели индивидуальные значения в каждой фазовой ячейке.

Для этого необходимо умножить все константы на специальные весовые („индивидуализирующие“) функции, значения которых остаются постоянными в пределах каждой фазовой ячейки, но меняются от ячейки к ячейке. При этом массивы констант и нелинейных 
членов в уравнениях (1) преобразуются к следующему виду:

$$
\begin{gathered}
\alpha_{\gamma}(x)=\left[\begin{array}{cccc}
\gamma_{\alpha 11}(x) \alpha_{11} & \gamma_{\alpha 12}(x) \alpha_{12} & \ldots & \gamma_{\alpha 1 n}(x) \alpha_{1 n} \\
\gamma_{\alpha 21}(x) \alpha_{21} & \gamma_{\alpha 22}(x) \alpha_{22} & \ldots & \gamma_{\alpha 2 n}(x) \alpha_{2 n} \\
\ldots & \ldots & \ldots & \ldots \\
\gamma_{\alpha n 1}(x) \alpha_{n 1} & \gamma_{\alpha n 2}(x) \alpha_{n 2} & \ldots & \gamma_{\alpha n n}(x) \alpha_{n n}
\end{array}\right] \\
f_{\gamma}[x, \eta]=\left[\begin{array}{c}
f_{1}\left[H_{1}\left(x_{1}\right), H_{2}\left(x_{2}\right), \ldots, H_{n}\left(x_{n}\right), \gamma_{\eta 11}(x) \eta_{11}, \gamma_{\eta 12}(x) \eta_{12}, \ldots, \gamma_{\eta 1 m_{1}}(x) \eta_{1 m_{1}}\right] \\
f_{2}\left[H_{1}\left(x_{1}\right), H_{2}\left(x_{2}\right), \ldots, H_{n}\left(x_{n}\right), \gamma_{\eta 21}(x) \eta_{21}, \gamma_{\eta 22}(x) \eta_{22}, \ldots, \gamma_{\eta 2 m_{2}}(x) \eta_{2 m_{2}}\right] \\
\ldots \\
f_{n}\left[H_{1}\left(x_{1}\right), H_{2}\left(x_{2}\right), \ldots, H_{n}\left(x_{n}\right), \gamma_{\eta n 1}(x) \eta_{n 1}, \gamma_{\eta n 2}(x) \eta_{n 2}, \ldots, \gamma_{\eta n m_{n}}(x) \eta_{n m_{n}}\right]
\end{array}\right]
\end{gathered}
$$

где $\gamma(x)-$ „индивидуализирующие“ функции, $x=x_{1}$, $x_{2}, \ldots, x_{n}$.

Следствием изменения констант в уравнениях движения является изменение параметров аттрактора, в том числе его конфигурации в фазовом пространстве системы. В частности, меняются размеры и взаимное положение фазовых ячеек, содержащих локальные аттракторы.

Совместить друг с другом такие ячейки без зазоров и перекрытий в общем случае невозможно. Поэтому после введения в уравнения весовых функций необходимо так изменить размеры и положение (относительно центров локальных систем координат) всех локальных аттракторов, чтобы содержащие их фазовые ячейки снова стали одинаковыми и приняли прежнее (такое же, как в однородном мультиаттракторе) упорядоченное расположение. (Для этого размеры всех локальных аттракторов и их положение внутри своих фазовых ячеек можно привести, например, к размерам и положению хаотического аттрактора исходной динамической системы.)

Нормирование размеров локальных аттракторов требует выполнить локальное преобразование масштаба фазового пространства в пределах каждой фазовой ячейки, т.е. в пределах $k$-й ячейки нужно сделать замену переменных вида

$$
x_{j}^{*}=\phi_{k, j} x_{j}, \quad j=1,2, \ldots, n,
$$

где $\phi_{k, j}-$ коэффициент преобразования масштаба в $k$-й ячейке по $j$-й переменной, что изменит размер аттрактора по новой переменной в $\phi_{k, j}$ раз.

Для приведения локальных аттракторов к одинаковому положению относительно центров локальных систем координат выражение (7) необходимо привести к виду

$$
x_{j}^{*}=\phi_{k, j}\left(x_{j}+\theta_{k, j}\right), \quad j=1,2, \ldots, n,
$$

где $\theta_{k, j}$ - интервал смещения $k$-го локального аттрактора по $j$-й переменной репликации.

Чтобы коэффициенты преобразования масштаба и симметрирующие коэффициенты имели индивидуальные значения в каждой фазовой ячейке, в уравнения движения необходимо ввести соответствующие масштабирующие и симметрирующие функции, обеспечивающие различные значения масштабных и симметрирующихкоэффициентов в пределах разных фазовых ячеек. Это можно сделать путем преобразования реплицирующих операторов к следующему виду

$$
H_{j}^{*}\left(x_{j}\right)=\phi_{j}(x) H\left(x_{j}+\theta_{j}(x)\right), \quad j=1,2, \ldots, n,
$$

где $\phi_{j}(x)$ и $\theta_{j}(x)-$ соответственно масштабирующая и симметрирующая функции по $j$-й переменной репликации. Значения данных функций, так же как значения весовых функций $\gamma(x)$, остаются постоянными в пределах каждой фазовой ячейки, но меняются от ячейки к ячейке.

После этого уравнения, описывающие динамику системы, имеющей композиционный хаотический мультиаттрактор, состоящий из неодинаковых локальных аттракторов, примут окончательный вид

$$
\dot{x}=\left[\begin{array}{c}
\dot{x}_{1}^{*} \\
\phi_{1}(x) \\
\dot{x}_{2}^{*} \\
\phi_{2}(x) \\
\cdots \\
\dot{x}_{n}^{*} \\
\phi_{n}(x)
\end{array}\right]
$$

где $\dot{x}_{j}=\frac{d x_{j}}{d t}, j=1,2, \ldots, n, \dot{x}^{*}=\alpha_{\gamma}(x) H^{*}(x)+f_{\gamma}(x, \eta)$,

$$
H^{*}(x)=\left[\begin{array}{c}
\phi_{1}(x) H_{1}\left[x_{1}+\theta_{1}(x)\right] \\
\phi_{2}(x) H_{2}\left[x_{2}+\theta_{2}(x)\right] \\
\cdots \\
\phi_{n}(x) H_{n}\left[x_{n}+\theta_{n}(x)\right]
\end{array}\right]
$$

$$
\alpha_{\gamma}(x)=\left[\begin{array}{cccc}
\gamma_{\alpha 11}(x) \alpha_{11} & \gamma_{\alpha 12}(x) \alpha_{12} & \ldots & \gamma_{\alpha 1 n}(x) \alpha_{1 n} \\
\gamma_{\alpha 21}(x) \alpha_{21} & \gamma_{\alpha 22}(x) \alpha_{22} & \ldots & \gamma_{\alpha 2 n}(x) \alpha_{2 n} \\
\ldots & \ldots & \ldots & \ldots \\
\gamma_{\alpha n 1}(x) \alpha_{n 1} & \gamma_{\alpha n 2}(x) \alpha_{n 2} & \ldots & \gamma_{\alpha n n}(x) \alpha_{n n}
\end{array}\right]
$$




$$
f_{\gamma}[x, \eta]=\left[\begin{array}{c}
f_{1}\left[H_{1}^{*}\left(x_{1}\right), H_{2}^{*}\left(x_{2}\right), \ldots, H_{n}^{*}\left(x_{n}\right), \gamma_{\eta 11}(x) \eta_{11}, \gamma_{\eta 12}(x) \eta_{12}, \ldots, \gamma_{\eta 1 m_{1}}(x) \eta_{1 m_{1}}\right] \\
f_{2}\left[H_{1}^{*}\left(x_{1}\right), H_{2}^{*}\left(x_{2}\right), \ldots, H_{n}^{*}\left(x_{n}\right), \gamma_{\eta 21}(x) \eta_{21}, \gamma_{\eta 22}(x) \eta_{22}, \ldots, \gamma_{\eta 2 m_{2}}(x) \eta_{2 m_{2}}\right] \\
\ldots \\
f_{n}\left[H_{1}^{*}\left(x_{1}\right), H_{2}^{*}\left(x_{2}\right), \ldots, H_{n}^{*}\left(x_{n}\right), \gamma_{\eta n 1}(x) \eta_{n 1}, \gamma_{\eta n 2}(x) \eta_{n 2}, \ldots, \gamma_{\eta n m_{n}}(x) \eta_{n m_{n}}\right]
\end{array}\right],
$$

Рассмотрим пример. Введем различия между локальными аттракторами в „Двумерном“ композиционном мультиаттракторе, состоящем из аттракторов Лоренца [14], существующем в следующей динамической системе [15]:

$$
\left\{\begin{array}{l}
\frac{d x}{d \tau}=A\left[H_{2}(y+\mu x)-(1+\mu) H_{1}(x)\right] \\
\frac{d y}{d \tau}=H_{1}(x)[B-z+\mu]-H_{2}(y+\mu x) \\
\frac{d z}{d \tau}=\left[H_{2}(y+\mu x)-\mu H_{1}(x)\right] H_{1}(x)-C z .
\end{array}\right.
$$

Репликация исходного аттрактора Лоренца в данной системе производится по двум переменным репликации: $x$ и $w=y+\mu x\left(\mu-\right.$ константа) операторами $H_{1}(x)$, $H_{2}(w)$.

Перед введением неоднородностей в мультиаттрактор системы (9) ее необходимо привести к виду (1), т.е. записать уравнения движения относительно переменных репликации:

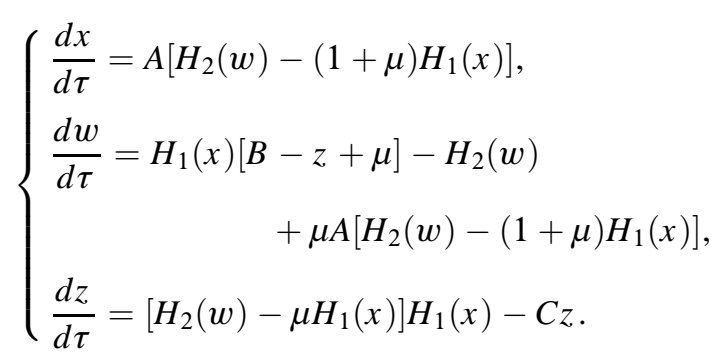

(Вследствие того, что в данной системе репликация по переменной $z$ не производится, оператор $H_{3}(z)$ является вырожденным: $H_{3}(z)=z$.)

Чтобы ввести различия между локальными аттракторами, присвоим константам $A, B, C$ индивидуальные значения в каждой фазовой ячейке. Для этого преобразуем уравнения (10) к виду

$$
\left\{\begin{array}{l}
\frac{d x}{d \tau}=\frac{\gamma_{A}(x, w) A 0\left[H_{2}^{*}(w)-(1+\mu) H_{1}^{*}(x)\right]}{\phi_{1}(x, w)}, \\
\frac{d w}{d \tau}=\frac{-H_{2}^{*}(w)+\mu \gamma_{A}(x, w) A\left[H_{2}^{*}(w)-(1+\mu) H_{1}^{*}(x)\right]}{\phi_{2}(x, w)} \\
\frac{d z}{d \tau}=\frac{-\gamma_{C}(x, w) C 0 \phi_{3}(x, w)\left(z+\theta_{3}(x, w)\right)}{\phi_{3}(x, w)}
\end{array}\right.
$$

где $A 0, B 0, C 0$ - значения констант, соответствующие аттрактору исходной динамической системы.
Так как аттрактор Лоренца симметричен по переменным $x$ и $w$, то соответствующие симметрирующие функции равны нулю: $\theta_{1}(x, w)=\theta_{2}(x, w)=0$, поэтому

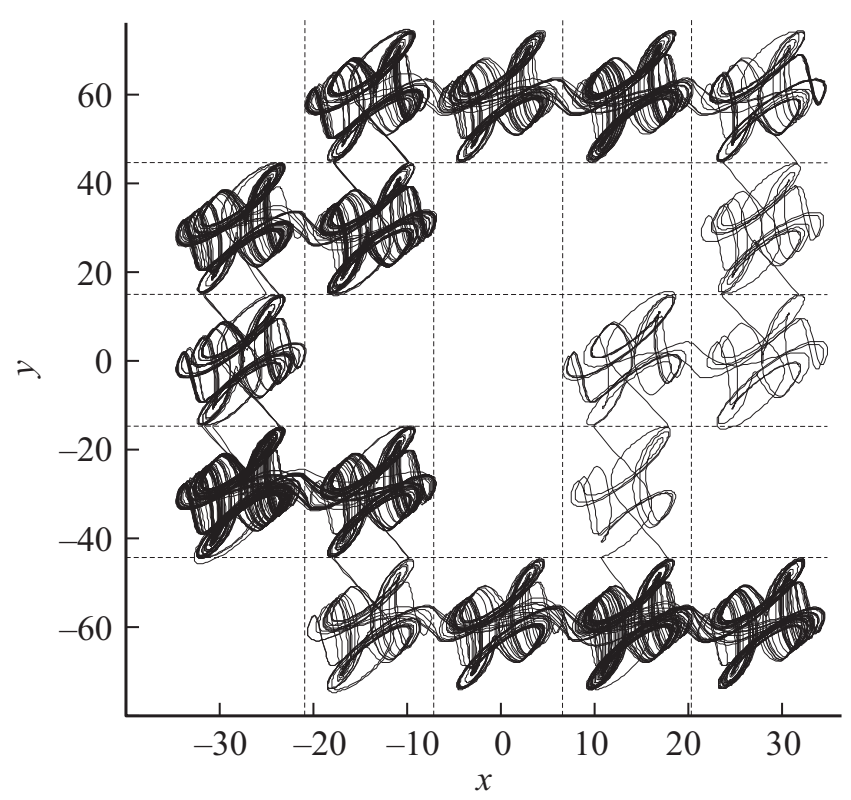

Рис. 4. Пример проекции на плоскость $(x, y)$ хаотического мультиаттрактора с заданной композиционной структурой, наблюдающегося в динамической системе (3), (4).

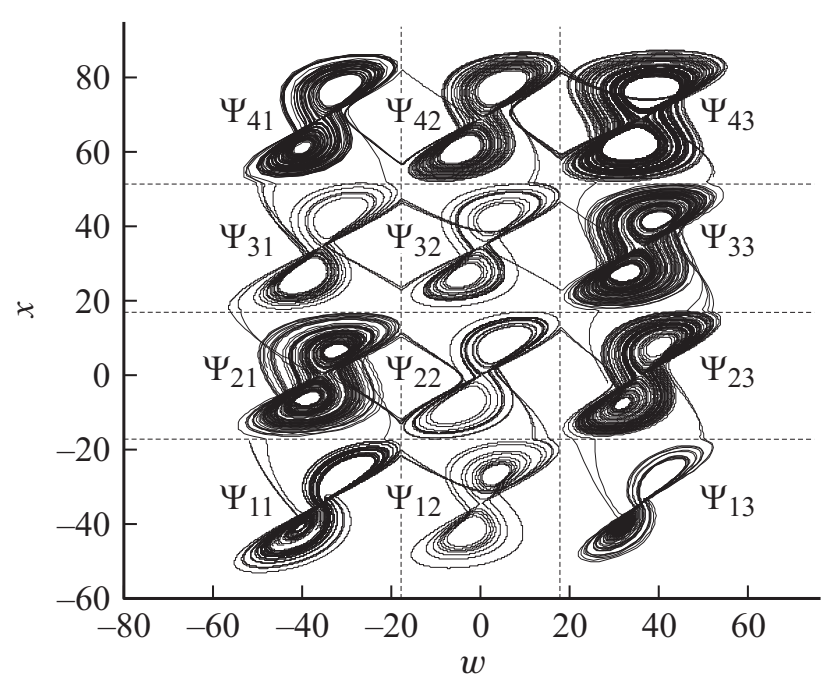

Рис. 5. Пример неоднородного составного хаотического мультиаттрактора, элементами которого являются аттракторы Лоренца, имеющие различные параметры. 
Таблица 1. Параметры уравнения (5), соответствующие функциям на рис. 2 и 3

\begin{tabular}{c|c|c|c|c|c|c|c|c}
\hline $\begin{array}{c}\text { Реплицирующий } \\
\text { оператор }\end{array}$ & \multicolumn{5}{|c|}{$H_{1}(x, y)$} & \multicolumn{4}{c}{$H_{2}(y, x)$} \\
\hline $\begin{array}{c}\text { Отклоняющая } \\
\text { функция }\end{array}$ & $\Delta m(y)_{1,1}$ & $\Delta m(y)_{1,2}$ & $\Delta n(y)_{1,1}$ & $\Delta n(y)_{1,2}$ & $\Delta m(x)_{2,1}$ & $\Delta m(x)_{2,2}$ & $\Delta n(x)_{2,1}$ & $\Delta n(x)_{2,2}$ \\
\hline$\xi$ & $y$ & $y$ & $y$ & $y$ & $x$ & $x$ & $x$ & $x$ \\
\hline$V_{1} ; W_{1} ; \Xi_{1}$ & $0 ; 0 ;-3 U_{2}$ & $0 ; 0 ;-3 U_{2}$ & $0 ; 0 ;-3 U_{2}$ & $R_{2} ; 3 ;-3 U_{2}$ & $0 ; 0 ;-3 U_{1}$ & $-R_{1} ;-3 ;-3 U_{1}$ & $0 ; 0 ;-3 U_{1}$ & $R_{1} ; 3 ;-3 U_{1}$ \\
\hline$V_{2} ; W_{2} ; \Xi_{2}$ & $-R_{2} ;-3 ;-2 U_{2}$ & $R_{2} ; 3 ;-2 U_{2}$ & $R_{2} ; 3 ;-2 U_{2}$ & $0 ; 0 ;-U_{2}$ & $-R_{1} ;-3 ;-2 U_{1}$ & $0 ; 0 ;-U_{1}$ & $R_{1} ; 3 ;-2 U_{1}$ & $0 ; 0 ;-U_{1}$ \\
\hline$V_{3} ; W_{3} ; \Xi_{3}$ & $0 ;-1 ; 2 U_{2}$ & $-R_{2} ;-1 ;-U_{2}$ & $0 ; 1 ; 2 U_{2}$ & $R_{2} ; 1 ; 0$ & $0 ;-1 ; 0$ & $-R_{1} ;-1 ; 0$ & $0 ; 1 ; 0$ & $R_{1} ; 1 ; 0$ \\
\hline$V_{4} ; W_{4} ; \Xi_{4}$ & $0 ; 0 ;-$ & $0 ; 0 ; U_{2}$ & $-R_{2} ; 3 ; 3 U_{2}$ & $-R_{2} ; 1 ; U_{2}$ & $0 ; 1 ; 2 U_{1}$ & $0 ;-1 ; 2 U_{1}$ & $0 ; 0 ; 3 U_{1}$ & $-R_{1} ; 1 ; U_{1}$ \\
\hline$V_{5} ; W_{5} ; \Xi_{5}$ & & $-R_{2} ; 1 ; 2 U_{2}$ & $0 ; 0 ;-$ & $0 ; 0 ; 3 U_{2}$ & $-R_{1} ; 3 ; 3 U_{1}$ & $R_{1} ;-3 ; 3 U_{1}$ & $-R_{1} ; 3 ; 4 U_{1}$ & $0 ; 0 ; 3 U_{1}$ \\
\hline$V_{6} ; W_{6} ; \Xi_{6}$ & & $R_{2} ;-3 ; 3 U_{2}$ & - & $-R_{2} ; 3 ;-$ & $0 ; 0 ;-$ & $0 ; 0 ; 3 U_{1}$ & $0 ;-1 ;-$ & $R_{1} ;-3 ; 4 U_{1}$ \\
\hline$V_{7} ; W_{7} ; \Xi_{7}$ & & $0 ; 0 ;-$ & - & - & - & & - & - \\
\hline
\end{tabular}

Таблица 2. Уравнения структурной функции

\begin{tabular}{|c|}
\hline$S(x, w, \Psi)=S 1(x, w, \Psi)+S 2(x, w, \Psi)+S 3(x, w, \Psi)$ \\
\hline $\begin{array}{c}S 1(t, q, \Psi)=\sum_{j_{2}=1-N_{2}}^{M_{2}-1} \sum_{j_{1}=1-N_{1}}^{M_{1}-1} \Psi_{j_{2}+O_{2}, j_{1}+O_{1}} P a\left[t-j_{1} c(1), q-j_{2} c(2)\right]+ \\
+\Psi_{j_{2}+O_{2}, M_{1}+O_{1}} P b 2\left[t-\left(M_{1}-1\right) c(1), q-j_{2} c(2)\right]+\Psi_{j_{2}+O_{2}, O_{1}-N_{1}} P b 4\left[t+\left(N_{1}-1\right) c(1), q-j_{2} c(2)\right]\end{array}$ \\
\hline $\begin{array}{c}S 2(t, q, \Psi)=\sum_{j_{1}=1-N_{1}}^{M_{1}-1} \Psi_{M_{2}+O_{2}, j_{1}+O_{1}} P b 1\left[t-j_{1} c(1), q-\left(M_{2}-1\right) c(2)\right]+ \\
+\Psi_{M_{2}+O_{2}, M_{1}+O_{1}} P c 1\left[t-\left(M_{1}-1\right) c(1), q-\left(M_{2}-1\right) c(2)\right]+\Psi_{M_{2}+O_{2}, O_{1}-N_{1}} P c 3\left[t+\left(N_{1}-1\right) c(1), q-\left(M_{2}-1\right) c(2)\right]\end{array}$ \\
\hline $\begin{array}{c}S 3(t, q, \Psi)=\sum_{j_{1}=1-N_{1}}^{M_{1}-1} \Psi_{O_{2}-N_{2}, j_{1}+O_{1}} P b 3\left[t-j_{1} c(1), q-\left(1-N_{2}\right) c(2)\right]+ \\
+\Psi_{O_{2}-N_{2}, M_{1}+O_{1}} P c 2\left[t-\left(M_{1}-1\right) c(1), q-\left(1-N_{2}\right) c(2)\right]+\Psi_{O_{2}-N_{2}, O_{1}-N_{1}} P c 4\left[t-\left(1-N_{1}\right) c(1), q-\left(1-N_{2}\right) c(2)\right],\end{array}$ \\
\hline $\begin{array}{c}P a(t, q)=P 3(t, 1) P 3(q, 2) ; P b 1(t, q)=P 3(t, 1) P 4(q, 2) ; P b 2(t, q)=P 4(t, 1) P 3(q, 2) \\
P b 3(t, q)=P 3(t, 1) P 5(q, 2) ; P b 4(t, q)=P 5(t, 1) P 3(q, 2) ; P c 1(t, q)=P 4(t, 1) P 4(q, 2) \\
P c 2(t, q)=P 4(t, 1) P 5(q, 2) ; P c 3(t, q)=P 5(t, 1) P 4(q, 2) ; P c 4(t, q)=P 5(t, 1) P 5(q, 2)\end{array}$ \\
\hline$P 3(\xi, \lambda)=P 1(\xi, \lambda)-P 2(\xi, \lambda), P 4(\xi, \lambda)=\frac{1}{2}+P 2(\xi, \lambda), P 5(\xi, \lambda)=\frac{1}{2}-P 1(\xi, \lambda)$ \\
\hline
\end{tabular}

Примечание: $M_{1}$ и $N_{1}$ - количество локальных аттракторов соответственно вверх и вниз по переменной $x$ от аттрактора исходной динамической системы, $M_{2}$ и $N_{2}$ - количество локальных аттракторов соответственно вверх и вниз по переменной $w$ от аттрактора исходной динамической системы, $h_{1}$ и $d_{1}-$ половина протяженности фазовой ячейки, содержащей хаотический аттрактор исходной динамической системы, и модуль крутизны промежуточных сегментов реплицирующей функции соответственно по переменной $x, h_{2}$ и $d_{2}-$ половина протяженности фазовой ячейки, содержащей хаотический аттрактор исходной динамической системы, и модуль крутизны промежуточных сегментов реплицирующей функции соответственно по переменной $w$.

в уравнениях (11)

$$
\begin{aligned}
H_{1}^{*}(x) & =\phi_{1}(x, w) H_{1}\left[x+\theta_{1}(x, w)\right]=\phi_{1}(x, w) H_{1}(x), \\
H_{2}^{*}(w) & =\phi_{2}(x, w) H_{2}\left[w+\theta_{2}(x, w)\right]=\phi_{2}(x, w) H_{2}(w) .
\end{aligned}
$$

Реплицирующие функции определяются уравнениями (2). Индивидуализирующие, масштабирующие и симметрирующие функции в случае двумерного композици- онного мультиаттрактора можно представить следующими выражениями:

$$
\begin{aligned}
\gamma_{A}(x, w)= & S(x, w, \gamma A), \gamma_{B}(x, w)=S(x, w, \gamma B), \\
& \gamma_{C}(x, w)=S(x, w, \gamma C), \\
\phi_{1}(x, w)= & S(x, w, \phi 1), \phi_{2}(x, w)=S(x, w, \phi 2),
\end{aligned}
$$




$$
\begin{gathered}
\phi_{3}(x, w)=S(x, w, \phi 3), \\
\theta_{1}(x, w)=S\left(x, w, \theta_{1}\right), \theta_{2}(x, w)=S\left(x, w, \theta_{2}\right), \\
\theta_{3}(x, w)=S\left(x, w, \theta_{3}\right),
\end{gathered}
$$

где $\gamma A, \gamma B, \gamma C$ - массивы значений весовых коэффициентов, задающих индивидуальные значения констант $A$, $B, C$, соответственно, в каждой фазовой ячейке; $\gamma \phi 1$, $\gamma \phi 2, \gamma \phi 3$ - массивы значений масштабных коэффициентов, обеспечивающих нормирование размеров всех локальных аттракторов по переменным $x, w, z$, соответственно; $\gamma \theta 1, \gamma \theta 2, \gamma \theta 3$ - массивы значений симметрирующих коэффициентов, обеспечивающих симметрирование локальных аттракторов по переменным $x, w, z$, соответственно.

$S(x, w, \Psi)$ - функция, задающая распределение коэффициентов по ячейкам фазового пространства; она представляет собой сумму составляющих, каждая из которых имеет заданное значение в пределах какой-то одной ячейки и равна нулю во всех остальных ячейках. Уравнения, определяющие эту функцию, приведены в табл. 2.

На рис. 5 приведен пример неоднородного хаотического мультиаттрактора, который наблюдается

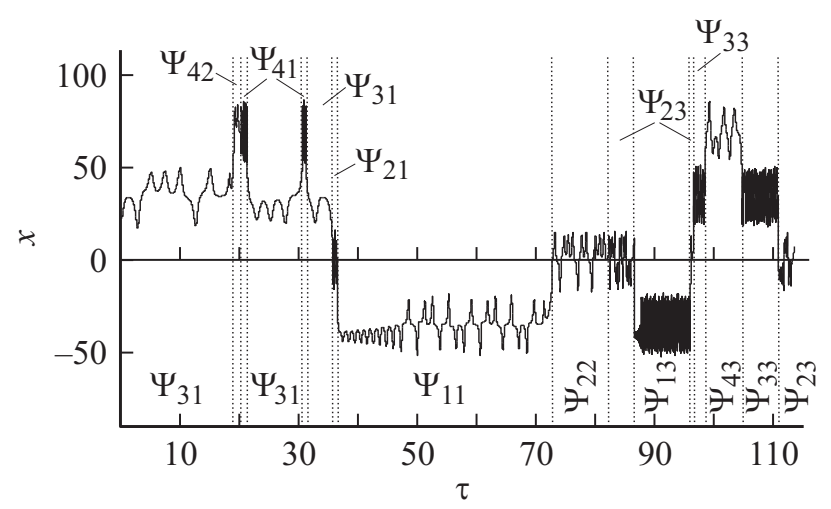

Рис. 6. Фрагмент временной зависимости переменной $x$. Показаны интервалы времени, соответствующие движению на различных локальных хаотических аттракторах.

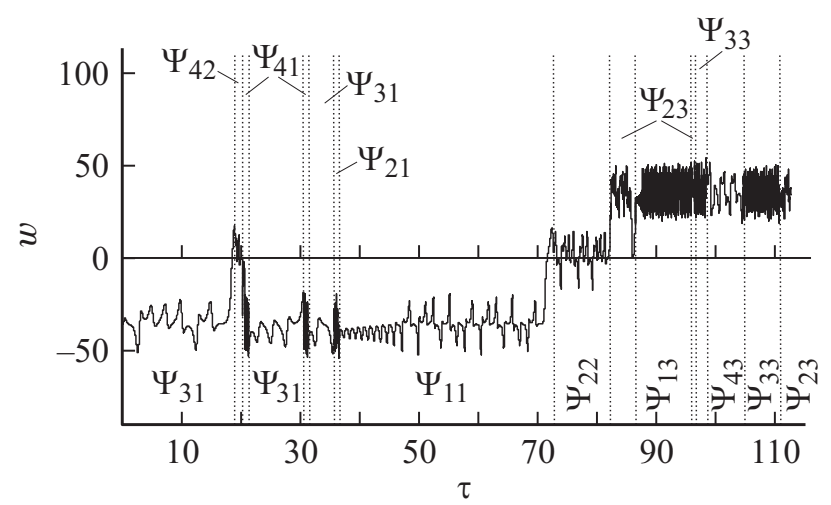

Рис. 7. Пример временно́й зависимости переменной $w$. Показаны интервалы времени, соответствующие движению на различных локальных хаотических аттракторах. в системе (11) при следующих значениях констант: $\mu=-0.45, \quad h_{1}=17.1, \quad h_{2}=17.7, \quad d_{1}=d_{2}=100, s_{1}=$ $=s_{2}=0, s_{3}=24.65, \quad A 0=10.5, \quad B 0=28, \quad C 0=8 / 3$, $M=2, \quad N=1, \quad M_{1}=2, \quad M_{2}=1, \quad N_{1}=N_{2}=1, \quad O_{1}=$ $=\mathrm{O}_{2}=2$.

$$
\begin{gathered}
\gamma A=\left[\begin{array}{cccc}
1.429 & 4.762 & 0.286 & 31.714 \\
0.19 & 1 & 0.381 & 1.429 \\
28.571 & 2.762 & 5.714 & 0.286
\end{array}\right], \\
\gamma B=\left[\begin{array}{cccc}
0.893 & 14.286 & 0.536 & 35.714 \\
0.714 & 1 & 0.714 & 1.786 \\
14.286 & 3.571 & 14.286 & 2.679
\end{array}\right], \\
\gamma C=\left[\begin{array}{cccc}
0.375 & 3.75 & 0.188 & 30 \\
0.113 & 1 & 0.375 & 1.5 \\
7.5 & 2.25 & 5.625 & 0.263
\end{array}\right], \\
\phi 1=\left[\begin{array}{cccc}
0.77 & 9 & 0.33 & 35 \\
0.31 & 1 & 0.55 & 1.6 \\
14.9 & 3.1 & 10 & 0.9
\end{array}\right], \\
\phi 2=\left[\begin{array}{cccc}
0.56 & 11 & 0.35 & 35 \\
0.4 & 0.9 & 0.6 & 1.6 \\
11.9 & 3.2 & 12.3 & 1.6
\end{array}\right], \\
\phi 3=\left[\begin{array}{cccc}
0.75 & 11.8 & 0.37 & 35 \\
0.4 & 1 & 0.63 & 1.65 \\
13.3 & 3.2 & 13.3 & 1.7
\end{array}\right], \\
{\left[\begin{array}{cccc}
5.5 & 9 & 11 & 2 \\
21 & 0 & 2.5 & 3 \\
4 & 5 & 4 & 17
\end{array}\right]} \\
\end{gathered}
$$

При этом сами константы имеют следующие значения:

$$
\begin{gathered}
A=\left[\begin{array}{cccc}
15 & 50 & 3 & 333 \\
2 & 10.5 & 4 & 15 \\
300 & 29 & 60 & 3
\end{array}\right], \\
B=\left[\begin{array}{cccc}
25 & 400 & 15 & 1000 \\
20 & 28 & 20 & 50 \\
400 & 100 & 400 & 75
\end{array}\right], \\
C=\left[\begin{array}{cccc}
1 & 10 & 0.5 & 80 \\
0.3 & 8 / 3 & 1 & 4 \\
20 & 6 & 15 & 0.7
\end{array}\right] .
\end{gathered}
$$

Все локальные аттракторы на рис. 5 приведены к одинаковым размерам, близким к размерам аттрактора исходной динамической системы (он расположен в фазовой ячейке, содержащей начало координат). На рис. 6 и 7 даны примеры временны́х зависимостей переменных $x$ и $w$. 


\section{Заключение}

Рассмотренные способы введения неоднородностей в композиционные хаотические мультиаттракторы состоят в изменении нелинейной части уравнений движения.

Формирование заданной композиционной структуры мультиаттракторов заключается в исключении из их состава части элементов. Это достигается путем такой модификации реплицирующих операторов, которая позволяет удалять часть ячеек фазового пространства, содержащих локальные аттракторы, за счет их поглощения расширенными соседними ячейками. Модификация реплицирующих операторов состоит во введении в их уравнения дополнительных членов, задающих локальное смещение границ между фазовыми ячейками относительно их положения, отвечающего однородному мультиаттрактору.

Для построения динамических систем, имеющих составные хаотические мультиаттракторы, в которых возможно априорное задание различий между входящими в их состав локальными хаотическими аттракторами, необходимо введение в уравнения движения трех групп дополнительных нелинейных функций, одна из которых отвечает собственно за введение различий между локальными аттракторами, а две другие - за нормирование размеров и конфигурации фазовых ячеек, содержащих локальные хаотические аттракторы.

\section{Список литературы}

[1] Lu J., Chen G. // Int. J. Bifurcat. Chaos. 2006. Vol. 16. N 4. P. 775-858.

[2] Strasser M., Theis F.J., Marr C. // Biophis. J. 2012. Vol. 102. P. 19-29.

[3] Luo X., Danca M.-F., Small M., Chen G. // Int. J. Bifurcat. Chaos. 2007. Vol. 17. N 9. P. 3235-3251.

[4] Yu S., Lu J., Chen G.// Phys. Lett. A. 2007. Vol. 364. P. 244 251.

[5] Munoz-Pacheco J.M., Tlelo-Cuautle E. // J. Appl. Res. Technol. 2009. Vol. 7. N 1. P. 5-13.

[6] Прокопенко В.Г. // Нелинейная динамика. 2012. Т. 8. № 3. C. 483-496.

[7] Прокопенко В.Г. Пат. РФ № 2207707. 2003, БИ 18.

[8] Прокопенко В.Г. // Докл. РАН. 2004. Т. 396. № 3. С. $317-$ 323.

[9] Прокопенко В.Г. // ЖТФ. 2013. Т. 83. Вып. 5. С. 6-9.

[10] Прокопенко В.Г. Пат. РФ № 2403672. 2010. БИ 31.

[11] Прокопенко В.Г. Пат. РФ № 2421877. 2011. БИ 17.

[12] Прокопенко В.Г. Пат. РФ № 2540817. 2015. БИ 4.

[13] Прокопенко В.Г. // Вестник МГТУ им. Н.Э. Баумана. Сер. Естественные науки. 2010. № 4. С. 106-119.

[14] Lorenz E.N. // J. Atmos. Sci. 1963. Vol. 20. P. 130-141.

[15] Прокопенко В.Г. // Вестник МГТУ им. Н.Э. Баумана. Сер. Естественные науки. 2013. № 1. С. 61-72. 